\title{
Connectivity-Based Parcellation of the Human Frontal Pole with Diffusion Tensor Imaging
}

\author{
Huaigui Liu, ${ }^{1 \star}$ Wen Qin, ${ }^{1 \star}$ Wei Li, ${ }^{1}$ Lingzhong Fan, ${ }^{2}$ Jiaojian Wang, ${ }^{3}$ Tianzi Jiang, ${ }^{2}$ and Chunshui Yu ${ }^{1}$ \\ ${ }^{1}$ Department of Radiology, Tianjin Medical University General Hospital, Tianjin 300052, China, ${ }^{2}$ Sino-French Laboratory for Computer Science, \\ Automation, and Applied Mathematics, Center for Computational Medicine, National Laboratory of Pattern Recognition, Institute of Automation, Chinese \\ Academy of Sciences, Beijing 100190, China, and ${ }^{3}$ Key Laboratory for NeuroInformation of the Ministry of Education, School of Life Science and \\ Technology, University of Electronic Science and Technology of China, Chengdu 625014, China
}

The human frontal pole (FP) approximately corresponds to Brodmann's area 10 and is a highly differentiated cortical area with unique cytoarchitectonic characteristics. However, its functional diversity is highly suggestive of the existence of functional subregions. Based on anatomical connection patterns derived from diffusion tensor imaging data, we applied a spectral clustering algorithm to parcellate the human right FP into orbital (FPo), lateral (FPl), and medial (FPm) subregions. This parcellation scheme was validated by corresponding analyses of the left FP and right FP in another independent dataset. Both visual observation and quantitative comparison of the anatomical connection patterns of the three FP subregions revealed that the FPo showed greater connection probabilities to brain regions of the social emotion network (SEN), including the orbitofrontal cortex, temporal pole, and amygdala, the FPl showed stronger connections to the dorsolateral prefrontal cortex of the cognitive processing network (CPN), and the FPm showed stronger connections to brain areas of the default mode network (DMN), including the anterior cingulate cortex and medial prefrontal cortex. We further analyzed the restingstate functional connectivity patterns of the three FP subregions. Consistent with the findings of anatomical connection analyses, the FPo was functionally correlated with the SEN, the FPl was correlated with the CPN, and the FPm was correlated with the DMN. These findings suggest that the human FP includes three separable subregions with different anatomical and functional connectivity patterns and that these subregions are involved in different brain functional networks and serve different functions.

\section{Introduction}

The frontal pole (FP) is the frontal part of the prefrontal cortex (PFC) and approximately corresponds to Brodmann's area (BA) 10, specifically area 10p of the human brain (Ongür et al., 2003; Ramnani and Owen, 2004). The human FP (area 10p) has unique and homogeneous cytoarchitectonic characteristics that distinguish it from the rest of the PFC (Ongür et al., 2003). Compared with the corresponding brain region in other mammals, the human FP has undergone great evolutionary expansion and exhibits a considerable increase in overall size and connections, especially with higher-order association areas (Semendeferi et al., 2001). Although data from humans are lacking, tracer studies in nonhuman primates have shown that the medial (FPm), lateral (FPl), and orbital (FPo) parts of the FP possess different anatomical

\footnotetext{
Received Oct. 15, 2012; revised March 2, 2013; accepted March 6, 2013.

Author contributions: H.L., W.Q., T.J., and C.Y. designed research; H.L., W.L., T.J., and C.Y. performed research; L.F. and J.W. contributed unpublished reagents/analytic tools; H.L., W.Q., W.L., L.F., J.W., and C.Y. analyzed data; H.L., T.J., and C.Y. wrote the paper.

This study was supported National Basic Research Program of China 973 Program Grant 2011CB707801 and Natural Science Foundation of China Grants 81271551, 81201152, and 81061120533.

*H.L. and W.Q. contributed equally to this work.

The authors declare no competing financial interests.

Correspondence should be addressed to either of the following: Chunshui Yu, Department of Radiology, Tianjin Medical University General Hospital, No. 154, Anshan Road, Heping District, Tianjin 300052, China, E-mail: chunshuiyu@yahoo.cn; or Tianzi Jiang, National Laboratory of Pattern Recognition, Institute of Automation, Chinese Academy of Sciences, Beijing 100190, China, E-mail: jiangtz@nlpr.ia.ac.cn.

DOI:10.1523/JNEUROSCI.4882-12.2013

Copyright $\odot 2013$ the authors $\quad 0270-6474 / 13 / 336782-09 \$ 15.00 / 0$
}

connection patterns (Petrides and Pandya, 2007; Burman et al., 2011), suggesting the possibility of parcellating the human FP based on anatomical connection patterns.

Despite its cytoarchitectonic homogeneity, the human FP has been shown to be a functionally heterogeneous region (Dumontheil et al., 2008). The human FP works together with the orbitofrontal cortex (OFC), temporal pole (TP), and amygdala (Amyg) to process social and emotional information (Olson et al., 2007), such as reward-related information (Koechlin and Hyafil, 2007; Boorman et al., 2009) and facial expression (Tsao et al., 2008). The FP also plays a critical role in many aspects of complex cognitive tasks (Burgess et al., 2006; Gilbert et al., 2006), including relational integration (Wendelken and Bunge, 2010), multitasking (Koechlin et al., 1999; Burgess et al., 2006; Medalla and Barbas, 2009), selection of processes or subgoals (Fletcher and Henson, 2001), and memory tasks (Ramnani and Owen, 2004; Badre and D'Esposito, 2009). Furthermore, the human FP is functionally correlated with the default mode network $(\mathrm{DMN})$, which is involved in internally focused tasks, such as prospecting the future (Schacter et al., 2007), autobiographical memory retrieval (Maguire et al., 2001), and envisaging the perspectives of others (Amodio and Frith, 2006). The functional diversity of the human FP suggests that it may consist of functional subregions, and this idea is supported by a meta-analysis of task activation that reported functional variation between FP1 and FPm (Gilbert et al., 2006b).

Diffusion tensor tractography (DTT) can show inter-regional anatomical connectivity in vivo and has been used extensively to 
parcellate heterogeneous brain regions based on their anatomical connection patterns (Behrens et al., 2003b). According to previous anatomical connection studies of the animal FP and functional studies of the human FP, we hypothesize here that the human FP includes at least three functionally independent subregions. We further hypothesize that each FP subregion has distinct anatomical and functional connectivity patterns and is involved in different brain functional networks. To test these hypotheses, we applied a DTT-based parcellation scheme to the human FP and studied the anatomical and functional connection patterns of each FP subregion from the perspective of functional networks.

\section{Materials and Methods}

Subjects and data acquisition. Two different datasets were obtained in this study. Dataset 1 was obtained from 12 healthy, right-handed subjects (five males; mean age, 25.5 years; range, 22-28 years), whereas dataset 2 was obtained from another cohort of eight healthy, right-handed subjects (three males; mean age, 22.3 years; range, 19-24 years). Dataset 1 included diffusion tensor imaging (DTI), structural MR imaging, and resting-state functional MRI (fMRI) data, whereas dataset 2 only included DTI with different scan parameters and structural MR imaging data. All MR images were acquired using a Signa HDx 3.0 tesla MR scanner with an eight-channel phased-array head coil. DTI data were acquired by a single-shot echo planar imaging sequence. The DTI parameters of dataset 1 were as follows: repetition time (TR), $15 \mathrm{~s}$; echo time (TE), $73 \mathrm{~ms}$; matrix, $128 \times 128$; field of view $(\mathrm{FOV}),=256 \times 256 \mathrm{~mm}^{2}$; slice thickness, $2 \mathrm{~mm}$ without gap; 69 axial slices; 50 non-collinear diffusion gradients $\left(b=1000 \mathrm{~s} / \mathrm{mm}^{2}\right)$ and three non-diffusion-weighted images $\left(b=0 \mathrm{~s} / \mathrm{mm}^{2}\right)$. Sagittal three-dimensional T1-weighted images were acquired by a brain volume sequence (TR, $7.8 \mathrm{~ms}$; TE, $3.0 \mathrm{~ms}$; FOV, $256 \times 256 \mathrm{~mm}^{2}$; matrix, $256 \times 256$; slice thickness, $1 \mathrm{~mm}$, no gap; 188 slices). The DTI parameters of dataset 2 were the same as for dataset 1 except for the following: TR, $10 \mathrm{~s}$; TE, $64.2 \mathrm{~ms}$; slice thickness, $3 \mathrm{~mm}$; 45 axial slices; and 55 diffusion gradients. The structural images of dataset 2 were the same as for dataset 1 except for the following: TR, 8.0; TE, $3.0 \mathrm{~ms}$; and 176 slices. The resting-state fMRI data of dataset 1 were obtained using a gradient-echo single-shot echo-planar imaging sequence with the following parameters: TR, 2000; TE, $30 \mathrm{~ms}$; slice thickness, $3 \mathrm{~mm}, 1 \mathrm{~mm}$ gap; matrix, $64 \times 64$; FOV, $240 \times 240 \mathrm{~mm}^{2}$; 38 transverse slices; 180 volumes. During fMRI scans, all subjects were instructed to keep their eyes closed, to stay as motionless as possible, to think of nothing in particular, and not to fall asleep. The study was approved by the Medical Research Ethics Committee of Tianjin Medical University, and all participants provided written informed consent forms.

Definition of seed and target masks. The human FP seed masks were extracted from the Harvard-Oxford cortical structural atlas (http://fsl. fmrib.ox.ac.uk/fsl/fslwiki/Atlases and http://www.cma.mgh.harvard. edu/manuals/parcellation) with a threshold of $50 \%$ minimum probability and wrapped back to the individual native DTI space using the inverse of linear transformation and nonlinear deformations. The FP seed mask was checked on the coronal planes slice by slice to include all tissue commonly assigned to the FP and to ensure that its posterior boundary was rostral to the anterior termination of the olfactory sulcus (Ramnani and Owen, 2004; John et al., 2007). In accordance with previous tract tracing (Petrides and Pandya, 2007; Burman et al., 2011) and anatomical studies (Ongür and Price, 2000; Kondo et al., 2003; Ongür et al., 2003) in nonhuman primates, six target brain regions (Table 1) in the same hemisphere were included in the FP connectivity fingerprints calculation (Lavenex and Amaral, 2000). The target regions were also extracted from the Harvard-Oxford cortical structural atlases using the same approach as for the extraction of the FP and subsequently transformed into individual diffusion space.

DTI data preprocessing. Both the DTI and T1-weighted data were visually inspected by two radiologists for apparent artifacts arising from subject motion and instrument malfunction. Distortions in the diffusion-weighted images caused by eddy currents and simple head motions were corrected by the Diffusion Toolbox of Functional MRI of the
Table 1. Target brain regions defined from previous tract tracing studies in nonhuman primates

\begin{tabular}{lll}
\hline Brain areas & Brodmann's areas & References \\
\hline OFC & BA 11-BA 14 & Burman et al., 2011; Ongür and Price, 2000; Ongür et al., 2003 \\
TP & BA 38 & Kondo et al., 2003; Burman et al., 2011 \\
Amyg & & Petrides and Pandya, 2007 \\
DLPFC & BA 8, BA 9, BA 46 & Petrides and Pandya, 2007; Burman et al., 2011 \\
ACC & BA 24, BA 32 & Petrides and Pandya, 2007; Burman et al., 2011 \\
MPFC & BA 9, BA 32 & Petrides and Pandya, 2007; Burman et al., 2011 \\
\hline
\end{tabular}

Brain (FMRIB) [FSL (FMRIB Software Library) 4.0; http://www.fmrib. ox.ac.uk/fsl]. Skull-stripped T1-weighted images were coregistered to the subject's non-diffusion-weighted images $\left(b=0 \mathrm{~s} / \mathrm{mm}^{2}\right)$ using statistical parametric mapping (SPM8; http://www.fil.ion.ucl.ac.uk/spm), which resulted in a set of coregistered T1 images in DTI space. The T1 images in diffusion space were then transformed to the Montreal Neurological Institute (MNI) space. Finally, the inverse transformation was used to transform the FP mask into individual diffusion space.

Probabilistic tractography. Tractography was performed in diffusion space using the FSL package. Voxelwise estimates of the fiber orientation distribution were performed using Bedpostx. We calculated probability distributions in two fiber directions at each voxel using multiple fiber extension (Behrens et al., 2007) based on a previously published diffusion modeling approach (Behrens et al., 2003a, b). We then estimated the fiber connections between each voxel in the seed region and every voxel of the whole brain. The connection probability between a seed and each of the other voxels in the brain is obtained by calculating the number of traces arriving at the target site. To reduce the false-positive connections, we thresholded the path distribution estimates from ProbTrack using a connection probability of $p<0.002$ (10 of 5000 samples). For data storage, all connectivity profiles of each voxel were downsampled to $5 \mathrm{~mm}$ isotropic voxels (Johansen-Berg et al., 2004). Cross-correlation between the connectivity patterns of all voxels in the seed mask were calculated and used for automatic parcellation (Johansen-Berg et al., 2004).

Tractography-based parcellation. The cross-correlation matrix was fed into a spectral clustering algorithm that used edge-weighted centroidal voronoi tessellations for image segmentation (Wang et al., 2009) for automatic clustering. The goal of clustering the cross-correlation matrix was to group together voxels of the seed region that share similar connection profiles with other voxels of the brain. However, the number of component clusters was chosen by the experimenter.

Selection of cluster number. To avoid arbitrary choice of the number of clusters, we used cross-validation to determine the number of clusters that yielded optimal consistency across subjects and, hence, the optimal number of clusters. Specifically, we used a leave-one-out method in which each subject's data were excluded from the averaging. For each subject, we checked the consistency between the clustering results of the single subject and the average across the remaining subjects using Cramer's $V$. Cramer's $V$ has values in the interval $[0,1]$, and high values indicate good consistency. A value of 1 indicates a perfect match. The intersubject consistency was checked for $k=2,3,4$, and 5 clusters.

Maximum probability map calculation. Considering inter-individual differences in the FP parcellation, we calculated the maximum probability map (MPM) to show the final results (Caspers et al., 2008). To do this, we transformed each individual parcellation result from diffusion space to the MNI template. The MPM was calculated in the MNI space by assigning each voxel to the subregion to which it was most likely to belong. For each FP subregion, we also calculated the probabilistic map and distributions of the probability values. The probability map and its corresponding probability distribution reflect the interindividual variability of each FP subregion.

Reproducibility. To validate the reliability of the spectral clustering method, we also conducted our parcellation scheme on the left FP. In addition, we recruited another independent dataset (dataset 2) with different scan parameters to validate the parcellation results of dataset 1.

Anatomical connectivity patterns of FP subregions. To show the different anatomical connection patterns of the FP subregions, the whole-brain 
probabilistic tractography for each subject was run from each FP subregion in individual diffusion space by estimating the fiber orientations in each voxel (Behrens et al., 2007). To highlight the interindividual variation, the fiber tracts were then warped into standard space, and an averaged fiber tract map was then computed for each FP subregion.

We also analyzed the probabilistic connections between each FP subregion and six predefined target regions (Table 1). For each FP subregion, we drew 5000 samples from the connectivity distribution of each voxel in the subregion and computed the probability of connections with all six target regions. The averaged connectivity probability values between each FP subregion and all the target regions were then calculated across all the subjects, and the results were used to generate anatomical connectivity fingerprints (Passingham et al., 2002).

Statistical analysis of connection probabilities was performed using SPSS 17.0 (SPSS). Repeated-measures ANOVA was performed to test the effects of hemispheres, FP subregions, and target regions on the connection probability values.

Resting-state fMRI data preprocessing. The resting-state fMRI data were preprocessed using SPM8 (http://www.fil.ion.ucl.ac.uk/spm) and DPARSF (Data Processing Assistant for Resting-State fMRI) (Chao-Gan and Yu-Feng, 2010). The first 10 volumes from each subject were discarded to allow the signal to reach equilibrium and the participants to adapt to the scanning noise. The remaining 170 volumes were corrected for acquisition time delay between slices. Then, head motion parameters were estimated; none of the subjects had a maximum displacement of $>1$ $\mathrm{mm}$ or a maximum rotation of $>1.0^{\circ}$. A unified segmentation approach was used to spatially normalize these functional images. The approach included the following steps: (1) individual structural images were coregistered to the mean functional image after motion correction; (2) the transformed structural images were segmented into gray matter, white matter, and CSF using a unified segmentation algorithm; and (3) the motion-corrected functional volumes were spatially normalized to MNI space using the normalized parameters estimated during segmentation, and functional images were then resampled into a voxel size of $2 \times 2 \times 2$ $\mathrm{mm}^{3}$. After normalization, images were smoothed using a Gaussian kernel of $6 \times 6 \times 6 \mathrm{~mm}^{3}$ full-width at half-maximum. Several sources of spurious variance, including the estimated motion parameters, the linear drift, and the average BOLD signals in the ventricular and white matter regions, were removed from the data through linear regression. Finally, temporal bandpass filtering $(0.01-0.08 \mathrm{~Hz})$ was performed on the time series of each voxel to reduce the effects of low-frequency drift and highfrequency noise. Four-dimensional residual time series data in the standard MNI space were acquired for each subject after the preprocessing.

Whole-brain resting-state functional connectivity pattern of each FP subregion. Each region of interest (ROI) of the FP subregion was defined as a sphere (radius, $6 \mathrm{~mm}$ ) centered at the peak coordinate of the probability map of each FP subregion. To ensure that all voxels of each ROI were within the gray matter, we multiplied each FP ROI by the gray matter mask. The mean time series of each FP ROI was then extracted from the four-dimensional residual time series data. For each subject, Pearson's correlation coefficients between the mean time series of each FP ROI and that of each voxel of the whole brain were computed and converted to $z$ values using Fisher's $r$-to- $z$ transformation to improve the normality. Then, individuals' $z$-values were entered into a random-effect onesample $t$ test in a voxelwise manner to identify brain regions that showed significant correlations with the seed ROI. Multiple comparisons were corrected for false discovery rate (FDR) with a threshold of $p<0.0001$ and a cluster size of $>100$ voxels. A paired $t$ test was used to identify the resting-state functional connectivity (rsFC) differences between every pair of FP subregions ( $p<0.05$, FDR corrected).

Comparison between anatomical and functional connectivity patterns of FP subregions. We calculated the ipsilateral rsFCs between each FP subregion and the six predefined target regions. Statistical analysis of rsFC strength was performed using SPSS 17.0 (SPSS). Repeated-measures ANOVA was performed to test the effects of hemispheres, FP subregions, and target regions on the rsFC strength. Finally, direct comparisons of anatomical and functional connectivity patterns were realized by fingerprint method.

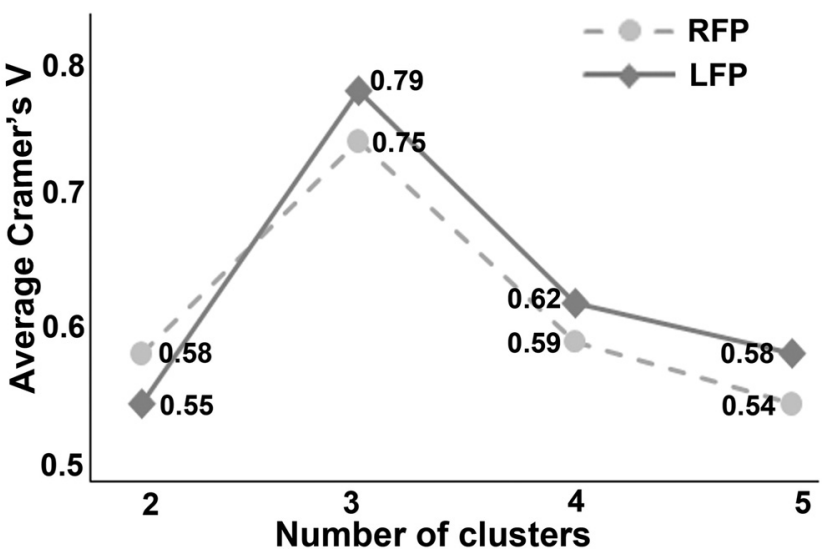

Figure 1. Average Cramer's $V$ as an indication of clustering consistency. Cramer's $V$ has values in the interval of $[0,1]$; high values indicate good consistency. It is shown that the three-cluster solution has the highest Cramer's V for both the left (LFP) and the right (RFP) FPs.

Reference to macaque data. Area 100 of the macaque is the equivalent area of the human FP (area 10p) (Ongür and Price, 2000). Although the area $10 \mathrm{o}$ of the macaque has expanded dramatically and evolved into the human area $10 \mathrm{p}$, we were interested in whether consistent connection patterns could be found in the corresponding areas of the two species. We summarized the anatomical connections of the area $10 \mathrm{o}$ reported in the previous tract tracer studies (Pandya and Seltzer, 1982; Morecraft et al., 2004) in the macaque based on the CoCoMac database (http://cocomac. org) (Stephan et al., 2001).

\section{Results}

\section{Connectivity-based parcellation}

In the spectral clustering method, it is important to select an optimal number of clusters. Although there is no gold standard for selecting the cluster number, we selected the optimal cluster number using a cross-validation method based on the consistency of clustering across subjects. We found that a cluster number of three gave the highest consistency of clustering across subjects (Fig. 1).

From the MPM of the right FP, we were able to identify three separable subregions of the FP: FPo, FPm, and FPl (Fig. 2A). This parcellation scheme was validated by similar analysis of the left FP (Fig. 2B) and the right FP in another independent dataset (Fig. $2 C)$. The probabilistic map of each FP subregion is shown in Figure $2 D$. The centriod distribution of each FP subregion across subjects is in Figure $3 A$. The averaged MNI coordinates of the centriods of the three FP subregions were as follows: FPo, left, $-22,62$, -9; right, 24, 62, -6; FPm, left, -10 , 64, 2; right, 9, 64, -1 ; and FPl left, $-20,61,17$; right, 20, 62, 16. To better visualize the location of each FP subregion, we also showed the FP subregions from different views (Fig. 3B).

\section{Anatomical connectivity patterns of FP subregions}

Using probabilistic tractography based on DTI data, fiber pathways of the three FP subregions were identified (Fig. 4). The fiber pathways predominantly followed the courses of the arcuate fibers, forceps minor, superior longitudinal fasciculus, cingulum, and uncinate fasciculus. To visualize the differential anatomical connection patterns of the three right FP subregions, we calculated the anatomical connections between each of the three FP subregions and the six predefined target regions. The six target regions were the OFC, Amyg, TP, dorsolateral PFC (DLPFC), anterior cingulate cortex (ACC), and medial PFC (MPFC) (Table 1). The right FPo showed stronger connection probabilities to 
A
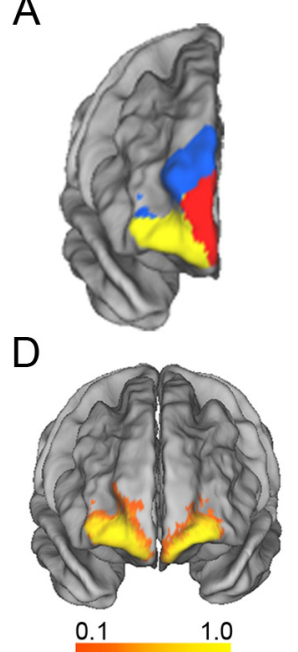

B
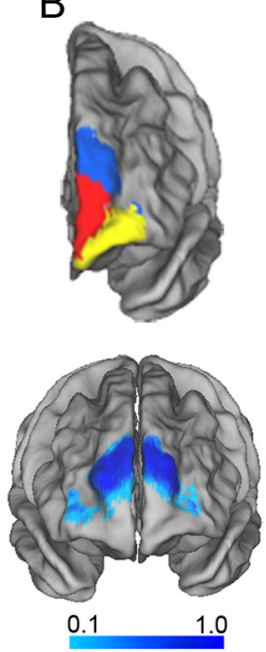

C
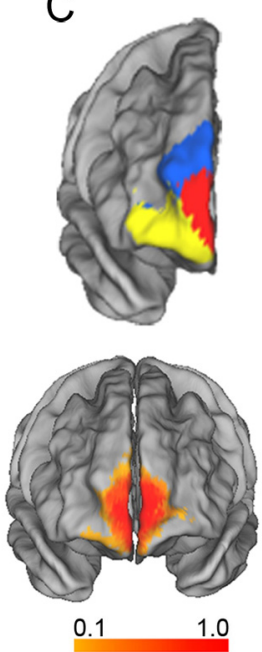

Figure 2. Connectivity-based parcellation of the human FP. The human FP can be reproducibly subdivided into orbital (yellow), lateral (blue), and medial (red) subregions, as shown in the maximum probabilistic maps of the right $(\boldsymbol{A})$ and left $(\boldsymbol{B}) \mathrm{FP}$ of dataset 1 and the right $\mathrm{FP}(\boldsymbol{C})$ of dataset 2. The probability map of each right FP subregion is shown in $\boldsymbol{D}$. The color bars represent the average probability across subjects at each voxel. Maps are displayed on a threedimensional brain surface using the Caret software.
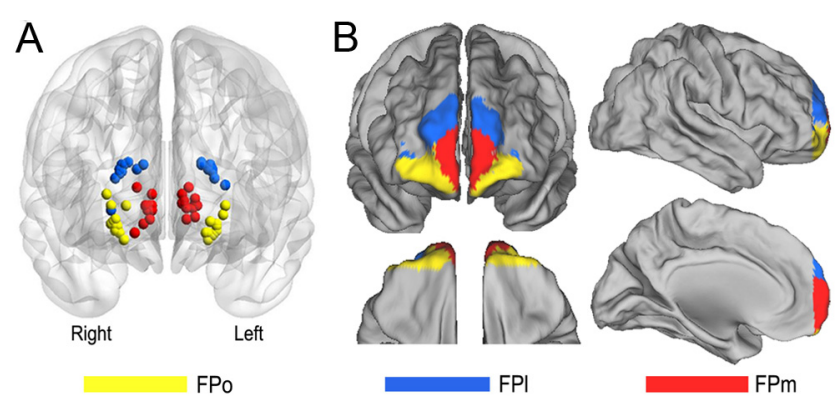

Figure 3. Centroid distribution $(\boldsymbol{A})$ and multiple views $(\boldsymbol{B})$ of the FP subregions. FP subregions are color coded as in Figure 2.

brain regions of the social emotion network (SEN), such as the OFC, Amyg, and TP. The right FPl showed greater connection probabilities to the right DLPFC, a critical node of the cognitive processing network (CPN). The right FPm showed stronger connection probabilities to brain areas of the DMN, including the ACC and MPFC.

\section{Quantitative comparisons of anatomical connections across the three FP subregions}

To quantify the differences in anatomical connections across the three FP subregions, the connection probabilities with the six target regions were normalized for the size of individual target ROIs (Table 2). Normalized connection probabilities were entered into a FP subregions (3 levels) $\times$ target regions ( 6 levels $) \times$ hemispheres (2 levels) repeated-measures ANOVA. There were no significant main effects in hemispheres $(F=0.143, p=0.705)$ and FP subregions $(F=0.142, p=0.868)$, but there was a significant main effect in target regions $(F=5.616, p<0.001)$. Moreover, there was a significant FP subregions $\times$ target regions interaction $(F=33.692, p<0.001)$. Post hoc comparisons were performed to visualize the anatomical connection probabilities differences across the three FP subregions (Table 3). The FPo showed higher probabilities of connection with the OFC, Amyg, and TP and lower probabilities of connection with the DLPFC

and ACC than the FPl. The FPl had a significantly higher probability of connection with the DLPFC and lower probabilities of connection with the ACC and MPFC than the FPm. The FPm showed higher probabilities of connection with the ACC and MPFC and lower probabilities of connection with the OFC, Amyg, and TP.

\section{Quantitative comparisons of rsFCs across the three FP subregions}

Using the same method as the quantitative comparisons of anatomical connections across the FP subregions, normalized rsFCs were also entered into the repeated-measures ANOVA model. There were no significant main effects in hemispheres $(F=0.036$, $p=0.849)$, but there were significant main effects in FP subregions $(F=25.143, p<0.001)$ and target regions $(F=20.937, p<$ $0.001)$. Moreover, there was a significant FP subregions $\times$ target regions interaction $(F=3.676, p<0.001)$. Post hoc comparisons were performed to visualize the rsFCs differences across the three FP subregions (Table 3). The FPl had a significantly higher rsFC with the DLPFC than the FPo and FPm. The FPm showed higher rsFCs with the ACC and MPFC than the FPo and FPl.

\section{Anatomical and functional connectivity fingerprints of FP subregions}

The fingerprint method was used to directly compare the anatomical and functional connectivity patterns of each FP subregion (Fig. 5). Generally, target regions that showed strong anatomical connections with an FP subregion also had strong rsFCs with that subregion, but the number of target regions with strong anatomical connections were much less than those that showed strong rsFCs. Specifically, although the FPo showed strong anatomical and functional connections with the TP and OFC, it only showed strong rsFCs with DLPFC and MPFC. The FPl showed higher anatomical and functional connections with the DLPFC; however, it also showed strong rsFCs with the other target regions. The FPm had strong anatomical and functional connections with the MPFC and ACC, whereas it only showed strong rsFC with the DLPFC.

\section{Whole-brain rsFC pattern of each FP subregion}

The whole-brain rsFC map of each FP subregion is displayed on the Caret PALS template (Van Essen et al., 2001; Van Essen, 2005) (Fig. 6). Overall, these three FP subregions showed different rsFC patterns, indicating that they are involved in different neural networks. The FPo was mainly correlated with the brain regions of the SEN, including the OFC and subgenual ACC $(p<0.0001$, FDR corrected). The FPl was mainly correlated with regions of the CPN, such as the DLPFC ( $p<0.0001$, FDR corrected). The FPm was correlated with the DMN, including the MPFC, posterior cingulate cortex/precuneus $(\mathrm{PCC} / \mathrm{Pcu})$ and lateral parietal cortex $(p<0.0001$, FDR corrected $)$.

To quantify the differences in rsFCs across the three FP subregions, the rsFCs were quantitatively compared ( $p<0.05$, FDR corrected) between every two FP subregions (Fig. 7). The FPo showed higher rsFC with the OFC and subgenual ACC and lower rsFC with the DLPFC than the FPl. The FPl had significantly higher rsFC with the DLPFC and lower rsFC with the ACC, MPFC, and PCC than the FPm. The FPm showed higher rsFC with the ACC, MPFC, and PCC and lower rsFC with the OFC.

\section{Reference to macaque data}

The anatomical connection patterns of area $10 \mathrm{o}$ in the macaque are shown in Figure 8. Area 10o in the macaque mainly connected 
to the OFC, ACC, MFPC, DLPFC, and TP, which is closely resemble those in the human FP (area 10p). However, rich connections with the Amyg were only found in human FP.

\section{Discussion}

To the best of our knowledge, this is the first study to parcellate the human FP based on anatomical connection patterns and to elucidate the anatomical and functional connectivity patterns of the human FP at the subregional level. The results show that the human FP can be reproducibly subdivided into $\mathrm{FPo}, \mathrm{FPl}$, and $\mathrm{FPm}$ subregions. Each FP subregion has specific anatomical and functional connectivity patterns, and the three subregions are involved in the SEN, CPN, and DMN, respectively. These findings may improve our understanding of FP connectivity and function at the level of subregions.

\section{Method consideration}

In the present study, we parcellated the human FP based on interregional anatomical connection patterns derived from DTT, which has been used extensively in previous parcellation studies of the human medial frontal cortex (Johansen-Berg et al., 2004), cingulate cortex (Beckmann et al., 2009), thalamus (Behrens et al., 2003b), and Amyg (Bach et al., 2011). However, one should bear in mind that the traditional DTT method is not the best one for accurately characterizing fiber directions (Jones et al., 2012); more plausible methods should be developed and used to parcellate human brain regions in vivo, such as parcellation based on the orientation distribution functions derived from the high angular resolution diffusion imaging data.

Spatial constraints have been used to reduce discontinuous voxels in parcellation results (Tomassini et al., 2007; Mars et al., 2012). Here, we did not place any spatial constraints on our parcellation scheme because the spectral clustering algorithm makes use of the eigenvectors of the similarity matrix as the feature for clustering, and this method is less sensitive to spatial distance effects than other clustering methods, such as the $k$-means algorithm (Ng et al., 2002). Moreover, how to balance connectivity information and spatial distance information in clustering when spatial constraints are included is still an open question. Here, we used MPMs to show the parcellation results for each FP subregion; this method could be effective in reducing discontinuous voxels with low probabilities. The spatially contiguous subregions also support the validity of our method.

\section{FP subregions}

In the present study, the human FP corresponds to area 10p, a highly differentiated cortical area with unique cytoarchitectonic characteristics (Ongür et al., 2003). However, as mentioned in Introduction, tract tracing and functional neuroimaging studies have suggested the existence of FP subregions. Ray and Price (1992) subdivided the rat FP into medial and lateral subregions based on thalamocortical connection patterns. The FP of nonhuman primates has been described as consisting of three subregions, $\mathrm{FPm}, \mathrm{FPl}$, and $\mathrm{FPo}$, based on their different anatomical connection patterns (Petrides and Pandya, 2007; Burman et al., 2011).

Compared with the animal FP, the human FP has undergone great evolutionary expansion and exhibits a lower cell density,
Table 2. Averaged normalized anatomical and functional connection strength between $F P$ subregions and target regions

\begin{tabular}{|c|c|c|c|c|c|c|}
\hline CD & OFC & Amyg & TP & DLPFC & $\mathrm{ACC}$ & MPFC \\
\hline subregions & $A C$ & $A C$ & $A C$ & $A C$ & $A C$ & $A C$ \\
\hline
\end{tabular}

$\begin{array}{lllllllllllllllll}\text { Right FPo } & 0.425 & 0.491 & 0.348 & 0.254 & 0.600 & 0.307 & 0.029 & 0.469 & 0.053 & 0.240 & 0.114 & 0.448\end{array}$ $\begin{array}{lllllllllllllllll}\text { Right FPI } & 0.018 & 0.627 & 0.108 & 0.331 & 0.160 & 0.452 & 0.580 & 0.853 & 0.274 & 0.472 & 0.317 & 0.765\end{array}$ Right FPm $0.098 \quad 0.4560 .0590 .3380 .0350 .4050 .127 \quad 0.606 \quad 0.5390 .574 \quad 0.6060 .893$ $\begin{array}{llllllllllllllllllllllll}\text { Left FPo } & 0.473 & 0.486 & 0.235 & 0.129 & 0.591 & 0.394 & 0.109 & 0.488 & 0.066 & 0.236 & 0.094 & 0.369\end{array}$ $\begin{array}{llllllllllllllllllllllll}\text { Left FPI } & 0.064 & 0.669 & 0.086 & 0.307 & 0.153 & 0.532 & 0.763 & 0.934 & 0.326 & 0.419 & 0.107 & 0.717\end{array}$ $\begin{array}{lllllllllllllllllllll}\text { Left FPm } & 0.094 & 0.449 & 0.055 & 0.306 & 0.102 & 0.401 & 0.069 & 0.622 & 0.598 & 0.553 & 0.662 & 0.873\end{array}$

$\mathrm{AC}$, Anatomical connection; $\mathrm{FC}$, functional connectivity.

richer dendritic spines, and more connections with the association cortices (Semendeferi et al., 2001). Gilbert et al. (2006b) found different activation characteristics for the lateral and medial portions of the human FP and further subdivided the human FP into lateral, anteromedial, and posteromedial subregions based on a meta-analysis of task activations (Gilbert et al., 2006). Based on anatomical connection patterns, we parcellated the human right FP into the orbital, medial, and lateral subregions and validated this parcellation scheme by parcellation of the left FP and through the use of another independent set of imaging data. The fact that our parcellation results are not completely consistent with those of Gilbert et al. (2006) can be ascribed to the differences in imaging measures, the FP definition, and parcellation methods between the two studies.

\section{Connectivity profiles of the FPo}

We found that the FPo is anatomically connected with the OFC, $\mathrm{TP}$, and Amyg and that it is functionally correlated with the OFC and subgenual ACC. The FPo connects with the OFC through the fronto-orbitopolar tract and with the TP and Amyg through the uncinate fasciculus, which are consistent with a DTT study in humans (Catani et al., 2012) and tract tracer studies in animals (Terreberry and Neafsey, 1987; Neafsey, 1990; Hurley et al., 1991). All of these brain regions are involved in the processing of social and emotional information. Each of these brain regions has been shown to be sensitive to different social or emotional tasks, and each of these tasks induced activation of different combinations of these brain regions. For example, the OFC is sensitive to reward-based decision making (Bechara et al., 1999; Boorman et al., 2009; FitzGerald et al., 2009; Elliott et al., 2010), the subgenual ACC is sensitive to negative emotional stimuli (Butler et al., 2005), the Amyg serves in the evaluation 
Table 3. Differences in the anatomical and functional connections between each pair of the FP subregions, shown with $p$ values (Bonferroni's corrected)

\begin{tabular}{|c|c|c|c|c|c|c|c|c|c|c|c|c|}
\hline \multirow[b]{2}{*}{ FP subregions } & \multicolumn{2}{|l|}{ OFC } & \multicolumn{2}{|l|}{ Amyg } & \multicolumn{2}{|l|}{ TP } & \multicolumn{2}{|l|}{ DLPFC } & \multicolumn{2}{|l|}{$\mathrm{ACC}$} & \multicolumn{2}{|l|}{ MPFC } \\
\hline & $\overline{A C}$ & $\mathrm{FC}$ & $A C$ & $\mathrm{FC}$ & $A C$ & $\mathrm{FC}$ & $A C$ & $\mathrm{FC}$ & $A C$ & $\mathrm{FC}$ & $A C$ & $\mathrm{FC}$ \\
\hline FPo versus FPI & $<0.001$ & 0.221 & $<0.001$ & 0.502 & $<0.001$ & 0.300 & $<0.001$ & $<0.001$ & 0.005 & 0.015 & 0.482 & $<0.001$ \\
\hline FPI versus FPm & 1.000 & 0.088 & 1.000 & 0.472 & 0.704 & 0.893 & $<0.001$ & 0.003 & 0.001 & 0.309 & $<0.001$ & 0.183 \\
\hline FPm versus FPo & $<0.001$ & 1.000 & $<0.001$ & 1.000 & $<0.001$ & 1.000 & 1.000 & 0.308 & $<0.001$ & $<0.001$ & $<0.001$ & $<0.001$ \\
\hline
\end{tabular}

AC, Anatomical connection; FC, functional connectivity.

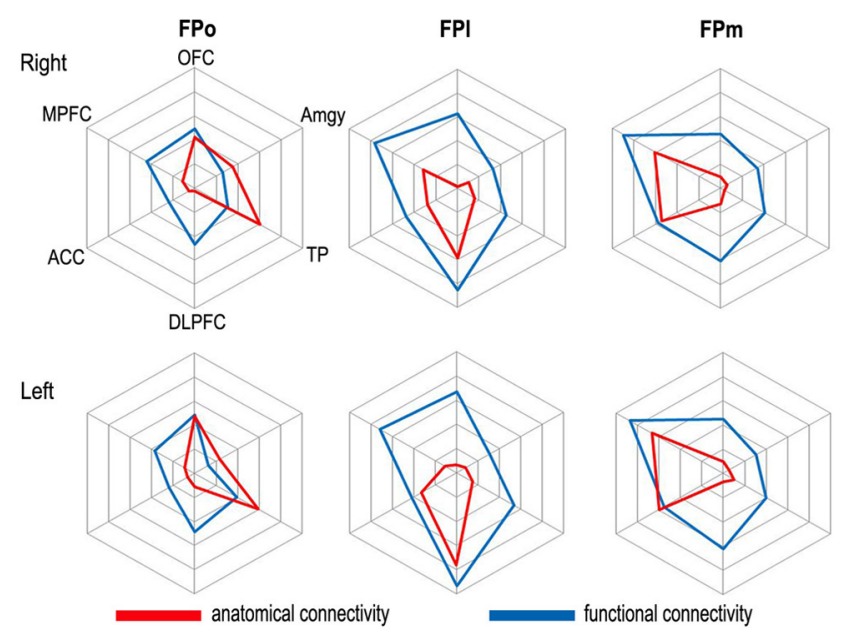

Figure 5. The comparisons between anatomical (red lines) and functional (blue lines) connectivity patterns of the FPo (left column), FPI (middle column), and FPm (right column) of the left (bottom row) and right (top row) hemispheres.

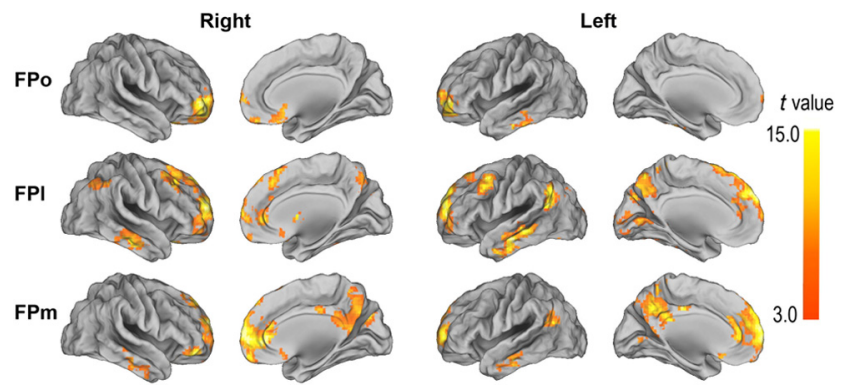

Figure 6. One-sample $t$ test shows the rsFC patterns of FP subregions $(p<0.0001$, FDR correction).

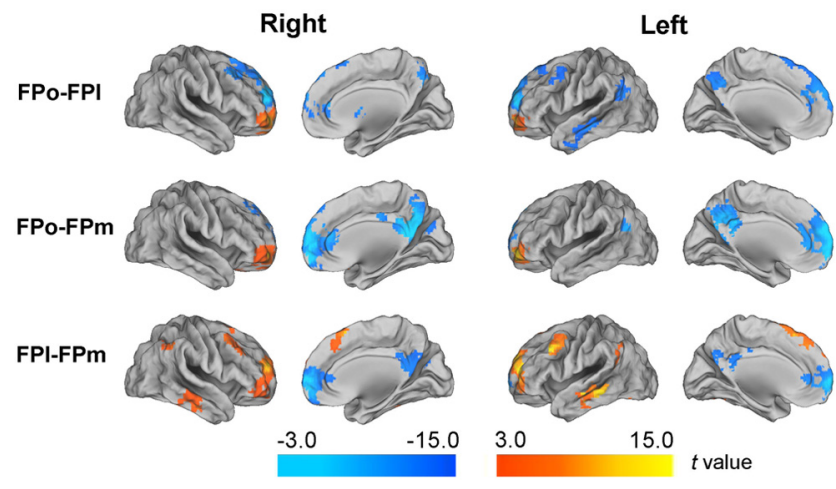

Figure 7. Contrast maps of the rsFCs between every two FP subregions.

of emotional information (Bechara et al., 1999; Winston et al., 2002), and both the TP and FPo are important for social and emotional processing (Olson et al., 2007; Zahn et al., 2009). Compared with the macaque FP, the human FPo had richer

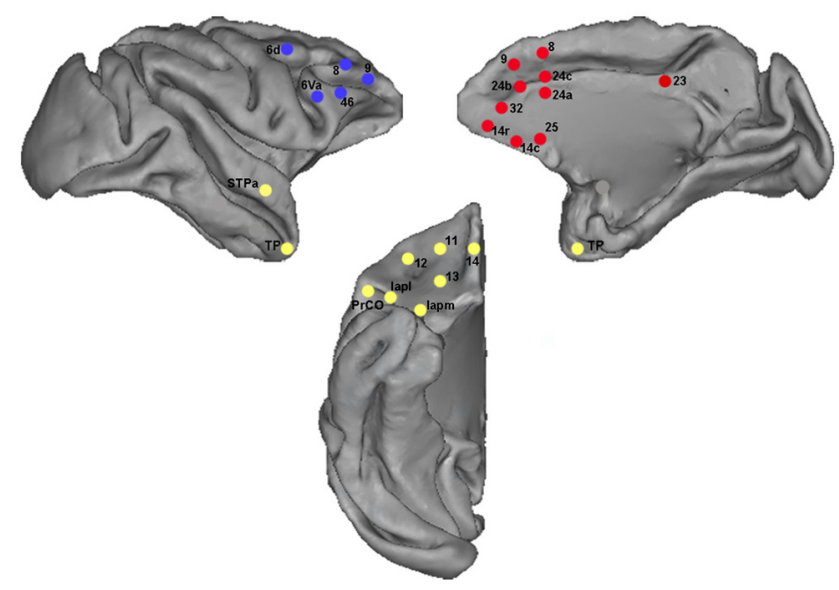

Figure 8. Schematic drawings of areas connected with area 100 in the macaque. Color dots indicate the presence of a connection with the lateral (blue), medial (red), and orbital (yellow) parts of the area 100. Connections depicted here were obtained from and named according to the CoCoMac database (http://cocomac.org).

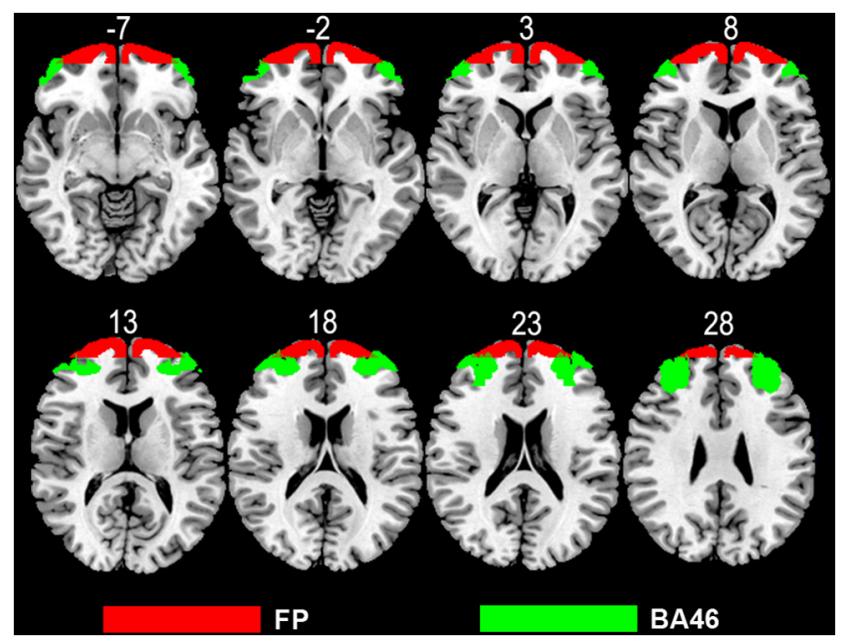

Figure 9. Overlap between the FP seed mask (red) and the BA 46 (green).

connections with the Amyg, which may underlie the complex emotion processing in human.

\section{Connectivity profiles of the FPl}

Anatomical and functional connectivity analyses have revealed that the FPl is closely associated with the immediately adjacent parts of the DLPFC, which is consistent with a human DTT study (Catani et al., 2012) and tracer studies in the macaque (Stephan et al., 2001). As shown in Figure 9, the lack of overlap between the seed FP and BA 46 (a portion of the DLPFC adjacent to the FP), suggesting that connection patterns of the FPl represent its unique connection patterns but not a reflection of the contamination of the BA 46. The strong connections between the FPl and DLPFC suggest that they are implicated in similar functions, such 
as working memory and episodic memory retrieval tasks (Fuster, 2001; Ranganath et al., 2003; Constantinidis and Procyk, 2004; Funahashi, 2006; Burgess et al., 2007; Cabeza and St Jacques, 2007; Koechlin and Hyafil, 2007; Smith et al., 2007; Badre and D'Esposito, 2009). The FP is particularly important in performing tasks that involve a high cognitive load (Fuster, 2001), such as relational integration (Wendelken and Bunge, 2010), multitasking (Koechlin et al., 1999; Burgess et al., 2006; Medalla and Barbas, 2009), and selection of processes or subgoals (Fletcher and Henson, 2001). Consistent with our viewpoint that the FPl is a critical node for cognitive processing, a meta-analysis showed that the FPl was disproportionately activated by cognitive tasks (Gilbert et al., 2006). Consistent with a finding that the human inferior parietal lobule (IPL) had functional connectivity with the FP that was not present in macaques (Mars et al., 2011), we also found the rsFC between the FPl and the central two IPL subregions (3 and 4), which are involved higher cognitive functions, such as exploratory decision (Daw et al., 2006; Boorman et al., 2009) and episodic memory retrieval (Velanova et al., 2003).

\section{Connectivity profiles of the FPm}

We found that the FPm is anatomically connected with the ACC and MPFC and that it is functionally correlated with the ACC, MPFC, PCC/Pcu, and lateral parietal cortex. The anatomical connections of the human FPm are also found in the macaque (Carmichael and Price, 1996; Ongür and Price, 2000; Petrides and Pandya, 2007), but they are not shown in a previous DTT study (Catani et al., 2012) because that study did not place an ROI in the FPm. The FPm, ACC, and MPFC are activated when individuals undertake internally focused tasks, including prospecting the future (Okuda et al., 2003; Addis et al., 2007; Schacter and Addis, 2007; Schacter et al., 2007; Sharot et al., 2007; Szpunar et al., 2007; Botzung et al., 2008), autobiographical memory retrieval (Maguire et al., 2001; Cabeza and St Jacques, 2007), selfrelated mentalizing (Gusnard et al., 2001; Frith and Frith, 2003; Amodio and Frith, 2006; Gilbert et al., 2006; Burgess et al., 2007; Raposo et al., 2011), and envisaging the perspectives of others (Saxe and Kanwisher, 2003; Amodio and Frith, 2006; Mitchell et al., 2006).

\section{Differences between anatomical and functional connection patterns of FP subregions}

We found that brain regions with strong anatomical connection with an FP subregion also had strong rsFC with that subregion, which is consistent with the statement that anatomical connection is the neural basis of rsFC (Petrides, 2005; Greicius et al., 2009). However, many brain areas only showed strong rsFCs with an FP subregion. These findings are consistent with previous observations (Eickhoff et al., 2010) and suggest that a very weak anatomical connection between two regions may still hold a high functional significance (Friston, 2002; Grefkes et al., 2008). It is more likely that the functional connectivity may reflect both direct and indirect anatomical connections between two brain regions (Honey et al., 2009).

\section{Conclusion}

In the present study, we found that the human FP can be divided into three subregions that show different anatomical and functional connection patterns. The three subregions are implicated in three parallel neural networks that process cognitive, selfreferential, and socio-emotional information, respectively. Notably, the human FP is the convergent node of these three networks, and the three FP subregions are closely connected with each other, which permits information exchange among these networks. The organization of the human FP thus supports its role in integrating various information sources to guide appropriate actions to a goal.

\section{References}

Addis DR, Wong AT, Schacter DL (2007) Remembering the past and imagining the future: common and distinct neural substrates during event construction and elaboration. Neuropsychologia 45:1363-1377. CrossRef Medline

Amodio DM, Frith CD (2006) Meeting of minds: the medial frontal cortex and social cognition. Nat Rev Neurosci 7:268-277. CrossRef Medline

Bach DR, Behrens TE, Garrido L, Weiskopf N, Dolan RJ (2011) Deep and superficial amygdala nuclei projections revealed in vivo by probabilistic tractography. J Neurosci 31:618-623. CrossRef Medline

Badre D, D'Esposito M (2009) Is the rostro-caudal axis of the frontal lobe hierarchical? Nat Rev Neurosci 10:659-669. CrossRef Medline

Bechara A, Damasio H, Damasio AR, Lee GP (1999) Different contributions of the human amygdala and ventromedial prefrontal cortex to decisionmaking. J Neurosci 19:5473-5481. Medline

Beckmann M, Johansen-Berg H, Rushworth MF (2009) Connectivity-based parcellation of human cingulate cortex and its relation to functional specialization. J Neurosci 29:1175-1190. CrossRef Medline

Behrens TE, Woolrich MW, Jenkinson M, Johansen-Berg H, Nunes RG, Clare S, Matthews PM, Brady JM, Smith SM (2003a) Characterization and propagation of uncertainty in diffusion-weighted MR imaging. Magn Reson Med 50:1077-1088. CrossRef Medline

Behrens TE, Johansen-Berg H, Woolrich MW, Smith SM, Wheeler-Kingshott CA, Boulby PA, Barker GJ, Sillery EL, Sheehan K, Ciccarelli O, Thompson AJ, Brady JM, Matthews PM (2003b) Non-invasive mapping of connections between human thalamus and cortex using diffusion imaging. Nat Neurosci 6:750-757. CrossRef Medline

Behrens TE, Berg HJ, Jbabdi S, Rushworth MF, Woolrich MW (2007) Probabilistic diffusion tractography with multiple fibre orientations: what can we gain? Neuroimage 34:144-155. CrossRef Medline

Boorman ED, Behrens TE, Woolrich MW, Rushworth MF (2009) How green is the grass on the other side? Frontopolar cortex and the evidence in favor of alternative courses of action. Neuron 62:733-743. CrossRef Medline

Botzung A, Denkova E, Manning L (2008) Experiencing past and future personal events: functional neuroimaging evidence on the neural bases of mental time travel. Brain Cogn 66:202-212. CrossRef Medline

Burgess PW, Simons JS, Dumontheil I, Gilbert SJ (2006) The gateway hypothesis of the rostral prefrontal cortex (area10) function. Oxford, UK: Oxford UP.

Burgess PW, Gilbert SJ, Dumontheil I (2007) Function and localization within rostral prefrontal cortex (area 10). Philos Trans R Soc Lond B Biol Sci 362:887-899. CrossRef Medline

Burman KJ, Reser DH, Yu HH, Rosa MG (2011) Cortical input to the frontal pole of the marmoset monkey. Cereb Cortex 21:1712-1737. CrossRef Medline

Butler T, Pan H, Epstein J, Protopopescu X, Tuescher O, Goldstein M, Cloitre M, Yang Y, Phelps E, Gorman J, Ledoux J, Stern E, Silbersweig D (2005) Fear-related activity in subgenual anterior cingulate differs between men and women. Neuroreport 16:1233-1236. CrossRef Medline

Cabeza R, St Jacques P (2007) Functional neuroimaging of autobiographical memory. Trends Cogn Sci 11:219-227. CrossRef Medline

Carmichael ST, Price JL (1996) Connectional networks within the orbital and medial prefrontal cortex of macaque monkeys. J Comp Neurol 371: 179-207. CrossRef Medline

Caspers S, Eickhoff SB, Geyer S, Scheperjans F, Mohlberg H, Zilles K, Amunts K (2008) The human inferior parietal lobule in stereotaxic space. Brain Struct Funct 212:481-495. CrossRef Medline

Catani M, Dell'acqua F, Vergani F, Malik F, Hodge H, Roy P, Valabregue R, Thiebaut de Schotten M (2012) Short frontal lobe connections of the human brain. Cortex 48:273-291. CrossRef Medline

Chao-Gan Y, Yu-Feng Z (2010) DPARSF: a MATLAB toolbox for "pipeline" data analysis of resting-state fMRI. Front Syst Neurosci 4:13. CrossRef Medline

Constantinidis C, Procyk E (2004) The primate working memory networks. Cogn Affect Behav Neurosci 4:444-465. CrossRef Medline

Daw ND, O’Doherty JP, Dayan P, Seymour B, Dolan RJ (2006) Cortical 
substrates for exploratory decisions in humans. Nature 441:876-879. CrossRef Medline

Dumontheil I, Burgess PW, Blakemore SJ (2008) Development of rostral prefrontal cortex and cognitive and behavioural disorders. Dev Med Child Neurol 50:168-181. CrossRef Medline

Eickhoff SB, Jbabdi S, Caspers S, Laird AR, Fox PT, Zilles K, Behrens TE (2010) Anatomical and functional connectivity of cytoarchitectonic areas within the human parietal operculum. J Neurosci 30:6409-6421. CrossRef Medline

Elliott R, Agnew Z, Deakin JF (2010) Hedonic and informational functions of the human orbitofrontal cortex. Cereb Cortex 20:198-204. CrossRef Medline

FitzGerald TH, Seymour B, Dolan RJ (2009) The role of human orbitofrontal cortex in value comparison for incommensurable objects. J Neurosci 29:8388-8395. CrossRef Medline

Fletcher PC, Henson RN (2001) Frontal lobes and human memory: insights from functional neuroimaging. Brain 124:849-881. CrossRef Medline

Friston K (2002) Functional integration and inference in the brain. Prog Neurobiol 68:113-143. CrossRef Medline

Frith U, Frith CD (2003) Development and neurophysiology of mentalizing. Philos Trans R Soc Lond B Biol Sci 358:459-473. CrossRef Medline

Funahashi S (2006) Prefrontal cortex and working memory processes. Neuroscience 139:251-261. CrossRef Medline

Fuster JM (2001) The prefrontal cortex-an update: time is of the essence. Neuron 30:319-333. CrossRef Medline

Gilbert SJ, Spengler S, Simons JS, Steele JD, Lawrie SM, Frith CD, Burgess PW (2006) Functional specialization within rostral prefrontal cortex (area 10): a meta-analysis. J Cogn Neurosci 18:932-948. CrossRef Medline

Gilbert SJ, Spengler S, Simons JS, Frith CD, Burgess PW (2006b) Differential functions of lateral and medial rostral prefrontal cortex (area 10) revealed by brain-behavior associations. Cereb Cortex 16:1783-1789. CrossRef Medline

Grefkes C, Eickhoff SB, Nowak DA, Dafotakis M, Fink GR (2008) Dynamic intra- and interhemispheric interactions during unilateral and bilateral hand movements assessed with fMRI and DCM. Neuroimage 41:13821394. CrossRef Medline

Greicius MD, Supekar K, Menon V, Dougherty RF (2009) Resting-state functional connectivity reflects structural connectivity in the default mode network. Cereb Cortex 19:72-78. CrossRef Medline

Gusnard DA, Akbudak E, Shulman GL, Raichle ME (2001) Medial prefrontal cortex and self-referential mental activity: relation to a default mode of brain function. Proc Natl Acad Sci U S A 98:4259-4264. CrossRef Medline

Honey CJ, Sporns O, Cammoun L, Gigandet X, Thiran JP, Meuli R, Hagmann P (2009) Predicting human resting-state functional connectivity from structural connectivity. Proc Natl Acad Sci U S A 106:2035-2040. CrossRef Medline

Hurley KM, Herbert H, Moga MM, Saper CB (1991) Efferent projections of the infralimbic cortex of the rat. J Comp Neurol 308:249-276. CrossRef Medline

Johansen-Berg H, Behrens TE, Robson MD, Drobnjak I, Rushworth MF, Brady JM, Smith SM, Higham DJ, Matthews PM (2004) Changes in connectivity profiles define functionally distinct regions in human medial frontal cortex. Proc Natl Acad Sci U S A 101:13335-13340. CrossRef Medline

John JP, Yashavantha BS, Gado M, Veena R, Jain S, Ravishankar S, Csernansky JG (2007) A proposal for MRI-based parcellation of the frontal pole. Brain Struct Funct 212:245-253. CrossRef Medline

Jones DK, Knosche TR, Turner R (2012) White matter integrity, fiber count, and other fallacies: the do's and don'ts of diffusion MRI. Neuroimage pii:S1053-8119(12)00730-6. CrossRef Medline

Koechlin E, Hyafil A (2007) Anterior prefrontal function and the limits of human decision-making. Science 318:594-598. CrossRef Medline

Koechlin E, Basso G, Pietrini P, Panzer S, Grafman J (1999) The role of the anterior prefrontal cortex in human cognition. Nature 399:148-151. CrossRef Medline

Kondo H, Saleem KS, Price JL (2003) Differential connections of the temporal pole with the orbital and medial prefrontal networks in macaque monkeys. J Comp Neurol 465:499-523. CrossRef Medline

Lavenex P, Amaral DG (2000) Hippocampal-neocortical interaction: a hierarchy of associativity. Hippocampus 10:420-430. CrossRef Medline

Maguire EA, Vargha-Khadem F, Mishkin M (2001) The effects of bilateral hippocampal damage on fMRI regional activations and interactions during memory retrieval. Brain 124:1156-1170. CrossRef Medline

Mars RB, Jbabdi S, Sallet J, O'Reilly JX, Croxson PL, Olivier E, Noonan MP, Bergmann C, Mitchell AS, Baxter MG, Behrens TE, Johansen-Berg H, Tomassini V, Miller KL, Rushworth MF (2011) Diffusion-weighted imaging tractography-based parcellation of the human parietal cortex and comparison with human and macaque resting-state functional connectivity. J Neurosci 31:4087-4100. CrossRef Medline

Mars RB, Sallet J, Schüffelgen U, Jbabdi S, Toni I, Rushworth MF (2012) Connectivity-based subdivisions of the human right "temporoparietal junction area": evidence for different areas participating in different cortical networks. Cereb Cortex 22:1894-1903. CrossRef Medline

Medalla M, Barbas H (2009) Synapses with inhibitory neurons differentiate anterior cingulate from dorsolateral prefrontal pathways associated with cognitive control. Neuron 61:609-620. CrossRef Medline

Mitchell JP, Macrae CN, Banaji MR (2006) Dissociable medial prefrontal contributions to judgments of similar and dissimilar others. Neuron 50: 655-663. CrossRef Medline

Morecraft RJ, Cipolloni PB, Stilwell-Morecraft KS, Gedney MT, Pandya DN (2004) Cytoarchitecture and cortical connections of the posterior cingulate and adjacent somatosensory fields in the rhesus monkey. J Comp Neurol 469:37-69. CrossRef Medline

Neafsey EJ (1990) Prefrontal cortical control of the autonomic nervous system: anatomical and physiological observations. Prog Brain Res 85:147165; discussion 165-166. Medline

Ng AY, Jordan MI, Weiss Y (2002) On spectral clustering: analysis and an algorithm. Adv Neural Inform Proc Syst 14:849-856.

Okuda J, Fujii T, Ohtake H, Tsukiura T, Tanji K, Suzuki K, Kawashima R, Fukuda H, Itoh M, Yamadori A (2003) Thinking of the future and past: the roles of the frontal pole and the medial temporal lobes. Neuroimage 19:1369-1380. CrossRef Medline

Olson IR, Plotzker A, Ezzyat Y (2007) The Enigmatic temporal pole: a review of findings on social and emotional processing. Brain 130:1718 1731. CrossRef Medline

Ongür D, Price JL (2000) The organization of networks within the orbital and medial prefrontal cortex of rats, monkeys and humans. Cereb Cortex 10:206-219. CrossRef Medline

Ongür D, Ferry AT, Price JL (2003) Architectonic subdivision of the human orbital and medial prefrontal cortex. J Comp Neurol 460:425-449. CrossRef Medline

Pandya DN, Seltzer B (1982) Intrinsic connections and architectonics of posterior parietal cortex in the rhesus monkey. J Comp Neurol 204:196210. CrossRef Medline

Passingham RE, Stephan KE, Kötter R (2002) The anatomical basis of functional localization in the cortex. Nat Rev Neurosci 3:606-616. Medline

Petrides M (2005) Lateral prefrontal cortex: architectonic and functional organization. Philos Trans R Soc Lond B Biol Sci 360:781-795. CrossRef Medline CrossRef Medline

Petrides M, Pandya DN (2007) Efferent association pathways from the rostral prefrontal cortex in the macaque monkey. J Neurosci 27:1157311586. CrossRef Medline

Ramnani N, Owen AM (2004) Anterior prefrontal cortex: insights into function from anatomy and neuroimaging. Nat Rev Neurosci 5:184-194. CrossRef Medline

Ranganath C, Johnson MK, D'Esposito M (2003) Prefrontal activity associated with working memory and episodic long-term memory. Neuropsychologia 41:378-389. CrossRef Medline

Raposo A, Vicens L, Clithero JA, Dobbins IG, Huettel SA (2011) Contributions of frontopolar cortex to judgments about self, others and relations. Soc Cogn Affect Neurosci 6:260-269. CrossRef Medline

Ray JP, Price JL (1992) The organization of the thalamocortical connections of the mediodorsal thalamic nucleus in the rat, related to the ventral forebrain-prefrontal cortex topography. J Comp Neurol 323:167-197. CrossRef Medline

Saxe R, Kanwisher N (2003) People thinking about thinking people. The role of the temporo-parietal junction in "theory of mind". Neuroimage 19:1835-1842. CrossRef Medline

Schacter DL, Addis DR (2007) The cognitive neuroscience of constructive memory: remembering the past and imagining the future. Philos Trans $\mathrm{R}$ Soc Lond B Biol Sci 362:773-786. CrossRef Medline

Schacter DL, Addis DR, Buckner RL (2007) Remembering the past to imag- 
ine the future: the prospective brain. Nat Rev Neurosci 8:657-661. CrossRef Medline

Semendeferi K, Armstrong E, Schleicher A, Zilles K, Van Hoesen GW (2001) Prefrontal cortex in humans and apes: a comparative study of area 10. Am J Phys Anthropol 114:224-241. CrossRef Medline

Sharot T, Riccardi AM, Raio CM, Phelps EA (2007) Neural mechanisms mediating optimism bias. Nature 450:102-105. CrossRef Medline

Smith R, Keramatian K, Christoff K (2007) Localizing the rostrolateral prefrontal cortex at the individual level. Neuroimage 36:1387-1396. CrossRef Medline

Stephan KE, Kamper L, Bozkurt A, Burns GA, Young MP, Kötter R (2001) Advanced database methodology for the Collation of Connectivity data on the Macaque brain (CoCoMac). Philos Trans R Soc Lond B Biol Sci 356:1159-1186. CrossRef Medline

Szpunar KK, Watson JM, McDermott KB (2007) Neural substrates of envisioning the future. Proc Natl Acad Sci U S A 104:642-647. CrossRef Medline

Terreberry RR, Neafsey EJ (1987) The rat medial frontal cortex projects directly to autonomic regions of the brainstem. Brain Res Bull 19:639649. CrossRef Medline

Tomassini V, Jbabdi S, Klein JC, Behrens TE, Pozzilli C, Matthews PM, Rushworth MF, Johansen-Berg H (2007) Diffusion-weighted imaging tractography-based parcellation of the human lateral premotor cortex identifies dorsal and ventral subregions with anatomical and functional specializations. J Neurosci 27:10259-10269. CrossRef Medline
Tsao DY, Schweers N, Moeller S, Freiwald WA (2008) Patches of faceselective cortex in the macaque frontal lobe. Nat Neurosci 11:877-879. CrossRef Medline

Van Essen DC (2005) A population-average, landmark- and surface-based (PALS) atlas of human cerebral cortex. Neuroimage 28:635-662. CrossRef Medline

Van Essen DC, Drury HA, Dickson J, Harwell J, Hanlon D, Anderson CH (2001) An integrated software suite for surface-based analyses of cerebral cortex. J Am Med Inform Assoc 8:443-459. CrossRef Medline

Velanova K, Jacoby LL, Wheeler ME, McAvoy MP, Petersen SE, Buckner RL (2003) Functional-anatomic correlates of sustained and transient processing components engaged during controlled retrieval. J Neurosci 23: 8460-8470. Medline

Wang J, Ju L, Wang X (2009) An edge-weighted centroidal Voronoi tessellation model for image segmentation. IEEE Trans Image Process 18: 1844-1858. CrossRef Medline

Wendelken C, Bunge SA (2010) Transitive inference: distinct contributions of rostrolateral prefrontal cortex and the hippocampus. J Cogn Neurosci 22:837-847. CrossRef Medline

Winston JS, Strange BA, O’Doherty J, Dolan RJ (2002) Automatic and intentional brain responses during evaluation of trustworthiness of faces. Nat Neurosci 5:277-283. CrossRef Medline

Zahn R, Moll J, Paiva M, Garrido G, Krueger F, Huey ED, Grafman J (2009) The neural basis of human social values: evidence from functional MRI. Cereb Cortex 19:276-283. CrossRef Medline 